\title{
Prevalência da alexitimia na anorexia nervosa e sua associação com variáveis clínicas e sociodemográficas
}

\author{
Prevalence of alexithymia in anorexia nervosa and its \\ association with clinical and sociodemographic variables
}

Sandra Torres', Marina Prista Guerra', Leonor Lencastre', Filipa Vieira', António Roma-Torres², Isabel Brandão²

\section{RESUMO}

\section{Palavras-chave}

Anorexia nervosa, sintomas afetivos, prevalência.

Objetivo: Analisar a prevalência da alexitimia numa amostra de pacientes com anorexia nervosa e sua relação com variáveis do foro clínico e sociodemográfico, em concreto, índice de massa corporal, duração da doença, idade, escolaridade e nível socioeconômico. Métodos: Foram avaliados 2 grupos de participantes do sexo feminino, com idades compreendidas entre os 13 e os 34 anos. Um grupo foi composto por 80 participantes com anorexia nervosa (Grupo AN) e o outro por 80 participantes saudáveis (Grupo Controle). A versão portuguesa da Toronto Alexithymia Scale - 20 items - foi aplicada a ambos os grupos. Resultados: A prevalência da alexitimia no Grupo AN foi 62,5\% e no Grupo Controle, $12,5 \%$. Os valores médios de alexitimia não diferiram significativamente entre os dois subtipos de AN, e ambos apresentaram valores estatisticamente superiores aos do Grupo Controle. A alexitimia não se correlacionou às variáveis clínicas e sociodemográficas consideradas, à exceção da escolaridade, cuja associação com a alexitimia foi positiva e baixa. Conclusão: Os pacientes com anorexia nervosa apresentaram, com elevada frequência, dificuldades na regulação dos afetos, independentemente de seu peso, tempo de evolução da doença, idade e nível socioeconômico. O tratamento deve privilegiar uma intervenção sistematizada no domínio das emoções.

\section{ABSTRACT}

Objective: To analyze the prevalence of alexithymia in anorexia nervosa and its relationship with clinical and sociodemographic variables, such as, body mass index, anorexia nervosa duration, age, years of education and socioeconomic status. Methods: Two groups of female participants, between the ages of 13 and 34 years, were recruited. One group was composed of 80 participants with anorexia nervosa (AN Group) and the other consisted of 80 healthy participants (Control Group). The Portuguese version of the Toronto Alexithymia Scale - 20 items - was applied to both groups. Results: The prevalence of alexithymia in the AN Group was 62.5\% and $12.5 \%$ in the Control Group. The mean values of alexithymia (total score and factors) did

1 Universidade do Porto, Faculdade de Psicologia e de Ciências da Educação, Porto, Portugal. 2 Hospital de São João, Serviço de Psiquiatria, Porto, Portugal. 


\section{Keywords}

Anorexia nervosa, affective symptoms, prevalence. not significantly differ between the two AN subtypes, and both subtypes presented higher statistical values when compared to the Control Group. Alexithymia did not correlate with clinical and sociodemographic variables, with the exception of years of education, which had a positive and low association with alexithymia. Conclusion: Patients with anorexia nervosa revealed a high prevalence of affect regulation deficits regardless of their weight, duration of the disease, age and socioeconomic status. Therapy should focus on a systematic intervention in the domain of emotional regulation.

\section{INTRODUÇÃO}

A anorexia nervosa (AN) é um transtorno alimentar (TA) que afeta principalmente mulheres jovens. Em seu desenvolvimento estão implicados fatores biológicos, psicológicos, familiares e socioculturais, bem como vulnerabilidades individuais a fatores de risco específicos ${ }^{1,2}$. No estudo dos fatores etiológicos, e não obstante o reconhecimento da natureza multifatorial destes, têm emergido com consistência posições teóricas que conceptualizam a AN como um transtorno na autorregulação, com especial predomínio dos déficits na regulação dos afetos ${ }^{3-5}$. Essa leitura teórica surgiu fortemente sustentada em descrições clínicas que afirmavam que indivíduos com AN vivenciavam suas emoções de uma forma confusa, manifestavam dificuldade em distingui-las e descrevê-las e, na sequência dessas dificuldades, evitavam diálogos que envolviam a comunicação de sentimentos ${ }^{6-8}$. Considerando a presença dessas características, o comportamento alimentar perturbado tem sido interpretado como um mecanismo regulador de estados emocionais intensos e negativos, desviando a atenção das emoções para o corpo e a alimentação $0^{9,10}$.

É com base nessa perspectiva que se tem desenvolvido o estudo da relação entre a AN e a alexitimia. A alexitimia é uma perturbação que afeta o processamento emocional, da qual resulta a incapacidade de exprimir as emoções, sob a forma de sentimentos, por intermédio da linguagem ${ }^{11}$. Suas características mais salientes são: (a) dificuldade em identificar e descrever sentimentos; (b) dificuldade em distinguir os sentimentos de sensações corporais decorrentes da atividade emocional; (c) processos imaginativos limitados; e (d) estilo cognitivo orientado para o exterior ${ }^{5,12}$. Estas duas últimas características são consideradas elementos básicos do pensamento operatório, evidenciando a pobreza ou a ausência de fantasias e a preocupação com detalhes pontuais de acontecimentos externos ${ }^{13}$.

Estudos realizados com indivíduos com AN têm encontrado uma elevada prevalência da alexitimia. Na revisão dos trabalhos publicados nesse domínio ${ }^{14-22}$, cujos resultados se apresentam com maior detalhe no Apêndice, verifica-se que a taxa de alexitimia frequentemente ascende a valores superiores a $50 \%$. Genericamente, tais valores podem ser consi- derados elevados, caso se tenha em conta que a prevalência da alexitimia na população geral varia entre 10\% e 20\%23,24 ou, de acordo com alguns estudos, até menos ${ }^{25-27}$. Adicionalmente, as análises comparativas efetuadas entre grupos de pacientes com AN e grupos de controle compostos por pessoas saudáveis têm reforçado a saliência de características alexitímicas nos indivíduos com AN9,16,18,19,21,22,28-31.

O que se desconhece, contudo, é se os dois subtipos de AN - tipo restritivo (AN-R) e tipo ingestão compulsiva/purgativo ou bulímico (AN-B) - contribuem de forma igualitária para essa elevada prevalência. Os estudos não são consensuais nos resultados apresentados, variando entre os que consideram que a expressão da alexitimia é distinta nos dois subtipos ${ }^{18,20}$ e os que não observaram diferenças significativas entre eles ${ }^{14,15,21,29}$.

Também incidindo sobre as diferentes dimensões do conceito da alexitimia se levantam dúvidas sobre se, na AN, todas apresentam valores elevados, ou se tal fato apenas ocorre em algumas. A presença acentuada da dificuldade em identificar sentimentos (Fator 1 da alexitimia - F1) e a dificuldade em descrever sentimentos aos outros (Fator 2 da alexitimia - F2) têm-se confirmado frequentemente em amostras de pacientes com AN, quando comparadas com grupos de pessoas saudáveis 16,18,21,22,31. Contudo, essa conclusão já não reúne consenso no que diz respeito ao estilo de pensamento orientado para o exterior (Fator 3 da alexitimia - F3). Na análise dessa dimensão, os estudos distinguem-se entre os que encontram valores mais elevados de F3 na AN18 e os que não encontram diferenças estatisticamente significativas em relação ao Grupo Controle 21,22,29,31. É de realçar que a análise da alexitimia tem sido predominantemente desenvolvida considerando seu nível global, sendo ainda escassos os trabalhos que, valorizando a natureza multidimensional dessa perturbação, têm analisado separadamente cada uma das suas dimensões.

Em síntese, é possível verificar que na AN há o predomínio de déficits na regulação dos afetos, todavia, existem várias especificidades na compreensão dessa relação que se encontram ainda por esclarecer, entre elas:

- se a alexitimia está associada à AN em geral ou se os dois subtipos de AN apresentam dificuldades em regular emoções em diferentes graus; 
- se todas as dimensões do conceito da alexitimia encontram expressão na $\mathrm{AN}$, ou se umas se evidenciam mais do que outras.

Adicionalmente a essas questões, levanta-se a hipótese de existirem algumas variáveis clínicas e sociodemográficas que possam potenciar a expressão das características alexitímicas. Em termos clínicos, é possível que a alexitimia possa variar em função do peso perdido no decurso da AN, sendo, dessa forma, uma consequência da restrição alimentar no funcionamento cognitivo. Os estudos mais recentes, que recorreram à medida mais comumente utilizada na avaliação dessa perturbação - a Toronto Alexithymia Scale (TAS-20) $)^{32}$-, oscilaram entre a ausência de uma relação significativa ${ }^{19} \mathrm{e}$ a presença de uma correlação negativa entre a alexitimia e o índice de massa corporal (IMC) ${ }^{18}$.

Para além do IMC, o tempo de duração da patologia alimentar tem sido igualmente ponderado como um possível fator influente na expressão da alexitimia, mas sem dados conclusivos. Råstam et al.19 deixaram em aberto essa hipótese ressaltando que a alexitimia pode ser mais prevalente durante a fase inicial da doença. Tal perspectiva não foi confirmada por Bourke et al. ${ }^{14}$, que não observaram diferenças significativas da alexitimia entre anoréticas com períodos de menor ou maior duração da doença.

No domínio das variáveis sociodemográficas, pondera-se a influência que a idade possa ter na presença da alexitimia, na medida em que as capacidades de regulação emocional se desenvolvem ao longo do tempo e podem ser influenciadas pela cultura geracional24,33. Com pacientes com TA, apenas se encontrou um estudo que analisou essa relação, tendo por conclusão que a idade não era um preditor significativo da alexitimia ${ }^{29}$.

Escassos são também os trabalhos que pesquisam na AN a associação entre as dificuldades de regulação emocional e a escolaridade. $O$ estudo dessa relação é pertinente na medida em que as dificuldades de comunicação podem influenciar o sucesso escolar e vice-versa ${ }^{27}$. Na AN, tanto quanto é do conhecimento do presente estudo, apenas Bourke et al. ${ }^{14}$ estudaram essa associação, encontrando uma correlação moderada e negativa entre a alexitimia e os anos de escolaridade.

O nível socioeconômico (NSE) é outra variável que pode influenciar a presença da alexitimia. Essa perturbação pode se desenvolver secundariamente como uma estratégia para lidar com as emoções negativas inerentes às dificuldades socioeconômicas ou ser ela própria um fator que dificulte o acesso a um NSE mais elevado, resultado de um menor sucesso na área social24,27. Com amostras de AN não se verificou nenhuma investigação que analisasse a relação entre as dificuldades de regulação emocional e a patologia alimentar.

Em conclusão, verifica-se que, apesar de haver uma forte evidência da presença de características alexitímicas na AN, ainda não é totalmente claro se essas dificuldades emocio- nais estão associadas a diferentes variáveis clínicas e sociodemográficas. Nesse sentido, este estudo visou dar um contributo para a compreensão das dificuldades de regulação emocional na AN, incidindo especialmente na análise das diferentes dimensões da alexitimia e de variáveis clínicas e sociodemográficas que poderão exercer influência na sua expressão. Especificamente pretendeu-se:

- definir a prevalência da alexitimia numa amostra de participantes com AN;

- analisar comparativamente os níveis da alexitimia e de seus fatores específicos, na AN-R, na AN-B e num grupo controle;

- avaliar se, na AN, os níveis de alexitimia variavam em função do IMC, da duração da doença, da idade, da escolaridade e do NSE.

\section{MÉTODOS}

\section{Participantes}

A amostra foi composta por 80 participantes, do sexo feminino, com AN (Grupo AN), que se encontravam em tratamento em 6 hospitais gerais e em 2 clínicas privadas. Relativamente ao subtipo de AN, a amostra teve a seguinte distribuição: 52 participantes com AN-R e 28 participantes com AN-B. A faixa etária dos participantes com AN variou entre os 13 e os 34 anos ( $M=19,21$; $D P=5,39)$. Como critério de inclusão para esse grupo, estabeleceu-se a presença do diagnóstico de AN, segundo os critérios definidos pelo Diagnostic and Statistical Manual of Mental Disorders - $4^{\text {th }}$ edition (DSM-IV) ${ }^{34}$. Foi considerado como critério de exclusão a coocorrência de dependência de álcool ou drogas, e de transtorno psicótico.

Foi ainda selecionado para este estudo um Grupo Controle, constituído por 80 participantes saudáveis, do sexo feminino e, em sua maioria, estudantes. As idades variaram entre os 13 e os 34 anos ( $M=19,20 ; D P=4,76)$. Apenas participantes sem diagnóstico atual ou prévio de TA e com peso normal, de acordo com a classificação da World Health Organization (IMC entre 18,5 e 24,9 kg/m²), foram incluídos no Grupo Controle. Esse grupo apresentou características sociodemográficas semelhantes ao grupo clínico no que concerne a sexo, idade, escolaridade e profissão.

\section{Instrumentos de medida}

Com o intuito de avaliar os critérios de inclusão foi aplicada, no Grupo AN, a Interview for the Diagnosis of Eating Disorders-IV (IDED-IV $)^{35}$, que permitiu confirmar o diagnóstico de AN de acordo com os critérios definidos no DSM-IV (APA, 1994), bem como recolher informações sociodemográficas (idade, anos de escolaridade, profissão do respondente e profissão dos pais). A versão portuguesa da IDED-IV apresentou propriedades válidas e fidedignas para ser utilizada enquanto 
medida de diagnóstico da AN ${ }^{36}$. Os participantes do Grupo Controle completaram um questionário inicial desenvolvido especialmente para este estudo e que visou avaliar: (a) a existência de história atual ou anterior de problemas de comportamento alimentar (Alguma vez Ihe foi diagnosticada um TA?); (b) presença de doenças (Tem algum tipo de doença, física ou mental?), (c) peso e altura, e (d) uso de medicação (Toma algum tipo de medicação?).

Para definição do NSE dos participantes do Grupo AN utilizou-se a categorização adaptada por Carmo et al. ${ }^{37}$, que constitui uma síntese da classificação nacional de profissões em cinco classes com as seguintes designações:

- classe 1: quadros superiores e especialistas das profissões intelectuais e científicas;

- classe 2: técnicos de nível médio e pessoal administrativo:

- classe 3: funcionários dos serviços;

- classe 4: trabalhadores manuais especializados;

- classe 5: trabalhadores manuais não especializados.

A classe 1 corresponde ao nível mais elevado e a classe 5, ao nível mais baixo. Registraram-se, ainda, as profissões e habilitações literárias do pai e da mãe, e considerou-se a profissão do membro que se situava no nível mais elevado como indicador da classe socioeconômica. Nos participantes não estudantes, isto é, já ativos em termos profissionais, não foi classificada a profissão dos pais, mas sim sua própria profissão. Os desempregados foram categorizados distintamente.

Para avaliar a alexitimia, os participantes de ambos os grupos preencheram a TAS-2032. A TAS-20 é um instrumento de autopreenchimento no qual os participantes são solicitados a marcar seu grau de concordância relativamente a cada um dos 20 itens, numa escala tipo Likert de 5 pontos, que oscilam entre "discordo totalmente" e "concordo totalmente". A TAS-20 foi adaptada para português por Prazeres et $a 1 .{ }^{38}$ e os resultados do estudo indicaram uma adequada consistência interna e uma estrutura fatorial em três fatores comparável à estrutura da versão original: (a) F1, dificuldade em identificar sentimentos; (b) F2, dificuldade em descrever sentimentos aos outros; e (c) F3, estilo de pensamento orientado para o exterior. Os pontos de corte propostos pelos autores e testados na amostra portuguesa indicam que escores totais iguais ou superiores a 61 revelam a presença de alexitimia. Os indivíduos que obtêm resultados iguais ou inferiores a 51 são considerados não alexitímicos e os que apresentam resultados entre 52 e 60 (inclusive) encontram-se numa zona charneira, isto é, intermediária entre as duas categorias, não podendo ser classificados nem como alexitímicos nem como não alexitímicos ${ }^{5}$.

\section{Procedimentos}

O estudo foi revisto e aprovado pela Universidade do Porto e foi obtida a aprovação ética nos hospitais e nas escolas. A participação no estudo foi voluntária. Os participantes do
Grupo AN assinaram, primeiramente, um Termo de Consentimento Livre e Esclarecido e, depois de o diagnóstico de AN ter sido confirmado por meio da aplicação da IDED-IV, completaram individualmente a TAS-20. No Grupo Controle, o questionário inicial e a TAS-20 foram aplicados coletivamente, durante o tempo de uma aula na instituição de ensino. Os participantes não estudantes foram selecionados segundo o método de amostragem por conveniência. Todos os participantes foram informados quanto aos objetivos do estudo e concordaram em participar.

\section{Análise de dados}

Foram efetuadas análises descritivas para definir a prevalência da alexitimia e foram aplicadas análises de variância univariada (ANOVA), com comparações post-hoc (teste de Tukey HSD), e análises de variância multivariada (MANOVA), com comparações post-hoc (teste de Scheffé), para comparar os níveis de alexitimia, e seus fatores, entre os grupos AN-R, AN-B e controle. Para explorar a relação da alexitimia com o IMC, duração da AN, idade e a escolaridade das anoréticas, procedeu-se ao cálculo do coeficiente de correlação $r$ de Pearson. Para analisar a correlação entre a alexitimia e o NSE, aplicou-se o coeficiente de correlação de Spearman.

Para todos os testes utilizados, foi considerado um nível de significância com valor $p<0,05$.

\section{RESULTADOS}

\section{Características clínicas e sociodemográficas}

Os pacientes do Grupo AN apresentaram uma duração mínima da doença de 3 meses e máxima de 10 anos ( $M=37,90$ meses; $D P=45,61)$. O IMC neste grupo variou entre 12,4 e 17,5 $\mathrm{kg} / \mathrm{m}^{2}\left(M=15,27 \mathrm{~kg} / \mathrm{m}^{2} ; \mathrm{DP}=1,49\right)$. Em termos sociodemográficos, o número de anos de escolaridade oscilou entre os 6 e os 17 anos ( $M=10,59 ; D P=2,68)$. Na análise da distribuição por classes socioeconômicas, consideraram-se as classes 1 e 2 como indicadoras de um NSE alto, a classe 3 como um nível intermediário, e as classes 4 e 5 como correspondentes a um nível baixo. Com base nessa classificação, verificou-se que $28,8 \%$ dos pacientes com AN enquadravam-se no NSE elevado, 30\% no NSE médio, e 32,5\% no NSE baixo.

No Grupo Controle, o IMC mínimo foi 19,0 e o máximo, $24,2 \mathrm{~kg} / \mathrm{m}^{2}\left(\mathrm{M}=21,08 \mathrm{~kg} / \mathrm{m}^{2} ; \mathrm{DP}=1,39\right)$. A escolaridade variou, igualmente, entre os 6 e os 17 anos $(M=11,38$; $D P=2,92)$.

\section{Prevalência da alexitimia e comparação entre grupos}

A presença da alexitimia foi determinada segundo os pontos de corte testados na amostra portuguesa. A análise de frequências comprovou que a prevalência de alexitimia no Grupo AN (considerando conjuntamente ambos os subtipos) 
foi de $62,5 \%$ em relação a 12,5\% no Grupo Controle. Especificamente no Grupo AN, detectou-se uma percentagem mais elevada de participantes alexitímicos no subtipo AN-R (67,3\%), comparativamente com o subtipo AN-B (53,6\%) (Tabela 1).

Tabela 1. Prevalência da alexitimia nos dois subtipos de anorexia nervosa e no Grupo Controle

\begin{tabular}{lccc}
\hline & $\begin{array}{c}\text { AN restritiva } \\
\mathbf{n}(\%)\end{array}$ & $\begin{array}{c}\text { AN bulimica } \\
\mathbf{n}(\%)\end{array}$ & $\begin{array}{c}\text { Controle } \\
\mathbf{n}(\%)\end{array}$ \\
\hline Alexitímicos & $35(67,3)$ & $15(53,6)$ & $10(12,5)$ \\
Zona intermediária & $8(15,4)$ & $10(35,7)$ & $18(22,5)$ \\
Não alexitímicos & $9(17,3)$ & $3(10,7)$ & $52(65,0)$ \\
Total & $52(100)$ & $28(100)$ & $80(100)$ \\
\hline
\end{tabular}

AN: anorexia nervosa.

Aplicou-se a ANOVA com o intuito de comparar os valores médios de alexitimia nos três grupos e verificou-se que existia uma diferença estatisticamente significativa entre eles, $F(2,155)=31,00(p<0,001)$. Comparações post-hoc (teste de Tukey HSD) revelaram que o escore médio da alexitimia na AN-R (M =63,81; DP = 10,86) foi estatisticamente superior ao do Grupo Controle ( $M=48,63 ; D P=12,30)$, com $p<$ 0,001. De igual forma, o Grupo AN-B $(M=63,07 ; D P=12,28)$ também apresentou um nível significativamente superior de alexitimia quando comparado com o Grupo Controle ( $p<$ $0,001)$. Ambos os subtipos de AN não diferiram significativamente entre si $(p=0,962)$.

Considerando a relevância de explorar detalhadamente os diferentes fatores da alexitimia, realizou-se uma análise de variância multivariada (MANOVA) para determinar o efeito do diagnóstico (AN-R, AN-B e Controle) nos três fatores da
TAS-20. Os resultados mostraram que ocorreram diferenças estatisticamente significativas entre os grupos na combinação dos três fatores: $F(6,302)=10,40(p<0,001)$; Wilks' Lam$\mathrm{bda}=0,69$; partial eta squared $=0,17$. As comparações post-hoc (teste de Scheffé) efetuadas com o intuito de identificar, para cada variável, as fontes das diferenças demonstraram que os participantes com AN-R e AN-B não apresentaram diferenças estatisticamente significativas entre si, mas ambos se distinguiram do Grupo Controle em todas as dimensões da alexitimia, indicando valores médios superiores, conforme apontado na tabela 2.

\section{Relação da alexitimia com variáveis clínicas e sociodemográficas}

O estudo dessa relação foi implementado especificamente no Grupo AN (considerando conjuntamente ambos os subtipos) e revelou que a alexitimia não se correlacionou significativamente com o IMC, a duração da doença e a idade dos participantes (Tabela 3). Essa conclusão aplica-se igualmente a cada um dos fatores da alexitimia. Apenas os anos de escolaridade se correlacionaram significativa e positivamente com o escore total da alexitimia e com F2, mesmo com valores baixos. Na análise da tabela de correlações verificou-se que os três fatores da alexitimia se correlacionaram significativamente entre si, com a exceção de F2 com F3, cujo valor obtido não foi estatisticamente significativo. A correlação de F1 com F3 foi baixa, ainda que estatisticamente significativa. No que concerne ao NSE dos participantes com AN, este não se correlacionou significativamente com o escore total da alexitimia $(\rho=-0,18 ; p=0,129)$, nem com F1 $(\rho=-0,14 ; p=$ $0,227)$, F2 $(\rho=-0,16 ; p=0,171)$ ou F3 $(\rho=-0,06 ; p=0,605)$.

Tabela 2. Diferenças nos três fatores da alexitimia em função do diagnóstico

\begin{tabular}{lccccc}
\hline & $\begin{array}{c}\text { AN restritiva }(\mathrm{n}=52) \\
\text { M (DP) }\end{array}$ & $\begin{array}{c}\text { AN bulímica }(\mathrm{n}=28) \\
\mathbf{M}(\mathrm{DP})\end{array}$ & $\begin{array}{c}\text { Controle }(\mathrm{n}=80) \\
\mathrm{M}(\mathrm{DP})\end{array}$ & F (df $=2)$ & Partial Eta Squared \\
\hline Alexitimia (F1) & $24,94(6,64)$ & $25,32(5,27)$ & $17,55(5,71)$ & $31,05^{* a, b}$ & 0,29 \\
Alexitimia (F2) & $17,75(4,36)$ & $17,29(5,06)$ & $13,33(4,65)$ & $16,59^{* a, b}$ & 0,18 \\
Alexitimia (F3) & $21,12(3,53)$ & $20,46(4,90)$ & $17,75(5,03)$ & $9,42^{* a, b}$ & 0,11 \\
\hline
\end{tabular}

a: diferenças significativas entre os grupos AN restritiva e controle; $b$ : diferenças significativas os grupos AN bulímica e controle; ${ }^{*} p<0,001$.

AN: anorexia nervosa; DP: desvio-padrão.

Tabela 3. Correlações entre a alexitimia (total e fatores), índice de massa corporal, duração da anorexia nervosa, escolaridade e idade, no grupo de pacientes com anorexia nervosa

\begin{tabular}{|c|c|c|c|c|c|c|c|}
\hline & Alexitimia (total) & Alexitimia (F1) & Alexitimia (F2) & Alexitimia (F3) & IMC & Duração AN & Idade \\
\hline Alexitimia (F1) & $0,86^{* * *}$ & & & & & & \\
\hline Alexitimia (F2) & $0,80^{* * *}$ & $0,59^{* * *}$ & & & & & \\
\hline Alexitimia (F3) & $0,57^{* * *}$ & $0,23^{*}$ & 0,22 & & & & \\
\hline IMC & $-0,08$ & $-0,12$ & $-0,16$ & 0,13 & & & \\
\hline Duração AN & $-0,10$ & $-0,10$ & 0,02 & $-0,15$ & $-0,17$ & & \\
\hline Idade & 0,03 & 0,08 & 0,05 & $-0,08$ & $0,23^{*}$ & $0,73^{* * *}$ & 0,11 \\
\hline Escolaridade & $0,22^{*}$ & 0,16 & $0,22^{*}$ & 0,13 & $-0,11$ & 0,07 & \\
\hline
\end{tabular}

IMC: índice de massa corporal; duração AN: tempo de duração da anorexia nervosa.

${ }^{* * *} p<0,001 ;{ }^{*} p<0,05$. 


\section{DISCUSSÃO}

Os dados deste estudo revelaram uma elevada prevalência da alexitimia em pacientes com AN (62,5\%). Confrontando, em específico, as taxas obtidas nos trabalhos realizados em outros países, conclui-se que em todos eles, e à semelhança de Portugal, os valores são superiores a 50\%. Contudo, alguns estudos apontaram taxas ligeiramente inferiores às obtidas na amostra portuguesa, com valores na ordem dos $55 \% 16,17$, e outros observaram valores superiores, por volta dos $70 \%^{14,22}$.

Considerando separadamente os dois subtipos, verificou-se que na AN-R a percentagem de alexitimia (67,3\%) foi ligeiramente superior à encontrada nos outros trabalhos, cujos valores oscilaram entre 56 e 63\% 15,18,20,21. Diferentemente, na $A N-B$, a prevalência $(53,6 \%)$ foi inferior à da maioria dos estudos ${ }^{15,18}$, cujos valores foram próximos dos $60 \%$. Salienta-se, no entanto, que o valor obtido para a AN-B foi muito semelhante ao observado por Speranza et al. ${ }^{21}$, com um número elevado de participantes com esse subtipo da doença.

Assim, e de uma forma geral, é possível considerar que a percentagem de casos de alexitimia identificados no presente estudo segue a tendência dos trabalhos realizados em outros países. As oscilações que se verificam entre as taxas calculadas nas diferentes investigações poderão estar associadas ao uso de diferentes versões e pontos de corte na escala de alexitimia, bem como a diferentes tamanhos das amostras (tendencialmente pequenos).

Neste trabalho, procurou-se ainda perceber se existiam diferenças consideráveis entre os níveis de alexitimia dos diferentes grupos e verificou-se que ambos os subtipos de AN apresentaram pontuações médias significativamente superiores ao Grupo Controle, tanto em relação ao escore total, como em cada um dos três fatores da alexitimia. Esse resultado em nível global de alexitimia tem sido bastante verificado em outros estudos 9,16,18,19,21,22,28-31. Todavia, a dúvida tem-se colocado em relação a F3, cujos valores médios nem sempre têm diferido significativamente entre amostras clínicas de TA e grupos de controle ${ }^{21,22,29,31}$. Porém, e neste ponto, o presente estudo revelou que os participantes com AN tenderam a utilizar um estilo de pensamento orientado para o exterior, diferenciando-se, assim, do Grupo Controle, à semelhança do que verificaram Eizaguirre et al. ${ }^{18}$.

Sendo a alexitimia um conceito multidimensional, tem-se colocado a hipótese de existirem diferenças etiológicas entre as três dimensões da alexitimia ${ }^{39}$, podendo F3 ser uma característica que se desenvolve com a acentuação das dificuldades em identificar e expressar emoções ${ }^{40}$ (F1 e F2), tornando-se estável e independente em casos de maior gravidade. O padrão de correlação de F3 com F1 e F2 aponta nesse sentido. Essa hipótese poderá justificar os diferentes resultados obtidos nos estudos anteriores, na medida em que as amostras poderão incluir pacientes com diferentes níveis de gravidade do TA.
Outro resultado que se realça da análise comparativa entre os grupos é a não diferenciação entre a AN-R e AN-B em relação à alexitimia (total e fatores). Essa conclusão difere da de Eizaguire et al. ${ }^{18}$ no que se refere ao resultado de F1, na medida em que esses autores observaram níveis mais elevados dessa dimensão na AN-B. Contudo, vale salientar que esse trabalho utilizou uma amostra mais reduzida de AN-R ( $n$ $=25)$ comparativamente com a do presente estudo $(n=52)$. Genericamente, os presentes resultados encontram paralelo nos de Bourke et al. ${ }^{14}$, Cochrane et al..$^{15}$, Speranza et al..$^{21}$ e Sexton et al. ${ }^{29}$, e sugerem que a alexitimia é uma característica relevante nas anoréticas em geral. Esse dado reforça a importância da intervenção psicológica orientada para a regulação emocional no âmbito do tratamento da AN.

Procurando perceber se a intervenção nessa área deverá equacionar o efeito de algumas variáveis clínicas (IMC e duração da doença) e sociodemográficas (idade, escolaridade e NSE), procedeu-se à análise das relações de cada uma delas com a alexitimia e verificou-se que, genericamente, a variação nos níveis de alexitimia (total e fatores) não se revelou associada a essas variáveis. A única exceção observou-se em relação à escolaridade que, apesar de ter significância estatística, apresentou valores de correlação baixos com o total da alexitimia e com F2.

Analisando especificamente as variáveis clínicas, o resultado desse estudo diferiu ligeiramente do obtido por Eizaguirre et al. ${ }^{18}$, que observaram uma correlação negativa e significativa com o IMC, apesar de ela ter uma força baixa. Comparando as características metodológicas dos dois trabalhos, é convicção deste estudo que o presente permite uma avaliação mais aprofundada na AN, na medida em que utiliza uma amostra composta unicamente por indivíduos com essa patologia específica. Contrariamente, a amostra considerada por Eizaguirre et al..$^{18}$ integrou também participantes com bulimia nervosa. É possível que existam especificidades na regulação emocional entre esses dois TA e, como tal, sugere-se que ambos sejam analisados separadamente.

A ausência de relação entre a alexitimia e o IMC diferenciou-se da tendência encontrada na maioria dos estudos com amostras comunitárias ${ }^{24,33,41}$ e sugere que essa dificuldade afetiva assume na AN um caráter central, estável e independente das variações de peso.

De igual forma, a alexitimia parece não variar em função do tempo de duração da patologia alimentar, à semelhança do que concluíram Bourke et al. ${ }^{14}$. Também esse dado reforça a perspectiva de que a alexitimia não é meramente uma adaptação ou uma complicação da AN.

A relativa estabilidade da alexitimia na AN é ainda reforçada pela ausência de associação com as variáveis sociodemográficas, idade e NSE. Nem as influências culturais e ambientais subjacentes às diferentes gerações, nem o NSE, ambos identificados nos estudos com amostras populacionais como estando relacionados à presença da alexitimia ${ }^{24,27,33,41}$, revela- 
ram-se determinantes na variação das características alexitímicas nos indivíduos com AN. De forma semelhante, o único estudo realizado no domínio dos TA concluiu que a idade não era um preditor significativo da alexitimia ${ }^{29}$.

A exceção surge relativamente à escolaridade, que apresentou uma correlação positiva e estatisticamente significativa com a alexitimia. No entanto, importa considerar que essa relação tem uma magnitude baixa $(r=0,22)$. Lane et al. ${ }^{33}$ chamaram a atenção para o fato de os indivíduos com pouca escolaridade poderem ter dificuldades na compreensão dos itens da escala de alexitimia. Ainda que a associação positiva entre alexitimia e os anos de escolaridade tenha sido pouco expressiva, pode-se colocar a hipótese de essa dificuldade emocional ter sido subavaliada nos participantes com menor nível educacional. Na opinião deste trabalho, e atendendo sobretudo à fraca magnitude dessa correlação, esse resultado aponta também no sentido de a escolaridade não exercer uma influência vincada na variabilidade dos níveis de alexitimia na AN.

Genericamente, conclui-se que a alexitimia é uma característica marcante nessa patologia e que parece não se desenvolver secundariamente como um efeito da doença, nem em associação a determinadas variáveis sociodemográficas. Essa estabilidade reforça a necessidade de o tratamento contemplar uma intervenção de base no domínio das emoções. Dessa abordagem poderão resultar consideráveis progressos terapêuticos em áreas que se estimam relacionadas com a dificuldade em reconhecer e comunicar emoções, tais como a insatisfação corporal, o sentido de ineficácia e os problemas na esfera interpessoal ${ }^{22,42}$.

Este trabalho apresenta algumas limitações que devem ser apontadas. A primeira decorre do uso de um instrumento de autorrelato para a avaliação da alexitimia, por si só dependente da consciência dos respondentes relativamente às suas capacidades no domínio emocional. Sugere-se que estudos futuros procurem complementar a avaliação da alexitimia com outras medidas que permitam a análise da experiência da emoção. Outra limitação está associada ao número desigual de participantes com AN-R e AN-B. Num estudo comparativo entre ambos os subtipos da doença seria desejável que as amostras tivessem um tamanho aproximado. Destaca-se ainda o fato de o NSE ter sido definido unicamente com base em critérios como a profissão e a escolaridade. Outros indicadores como os rendimentos e circunstâncias materiais (por exemplo, carro e habitação) permitiriam certamente uma definição mais precisa do NSE.

Para melhor clarificar a relação entre a alexitimia e a escolaridade, cujos resultados revelaram uma correlação positiva baixa, sugere-se que futuros trabalhos integrem outras variáveis associadas ao nível educacional, tal como a inteligência. O estudo da associação com o NSE seria também mais enriquecido com uma metodologia de investigação desenvolvimental e longitudinal. No domínio clínico, um aspecto relevante e que não foi contemplado neste estudo foi a ponderação da severidade da AN. A análise dessa variável permitiria perceber se existem variações nas dificuldades emocionais em função da gravidade da doença, dado esse que não é passível de ser obtido unicamente a partir da análise do IMC. Este estudo permitiria ainda aprofundar o conhecimento sobre a forma como F3 se relaciona com as outras dimensões da alexitimia.

\section{CONCLUSÕES}

A análise da prevalência de alexitimia e o estudo comparativo entre os grupos sugerem, conjuntamente, que a alexitimia é uma característica saliente na AN. As dificuldades na regulação dos afetos, uma vez desenvolvidas, parecem manifestar-se com alguma estabilidade nessa patologia, não variando em função do peso, tempo de duração da doença, ou variáveis sociodemográficas, como a idade e o NSE. Esses dados realçam a pertinência da abordagem emocional, num sentido transversal e continuado, no tratamento da AN.

\section{REFERÊNCIAS}

1. Cooper PJ. The development and maintenance of eating disorders. In: Brownell KD, Fairburn CG, editors. Eating disorders and obesity. New York: The Guilford Press; 1995. p. 199-206.

2. Garfinkel PE, Garner DM. Anorexia nervosa: a multidimensional perspective. New York: Brunner/Mazel; 1982.

3. Casper RC. Some provisional ideas concerning the psychologic structure in anorexia nervosa and bulimia. In: Darby PL, Garfinkel PE, Garner DM, Coscina DV, editors. Anorexia nervosa: Recent developments in research. New York: Alan R. Liss, 1983. p. 387-92.

4. Goodsitt A. Self-regulatory disturbances in eating disorders. Int J Eat Disord. 1993;2:51-60.

5. Taylor GJ, Bagby RM, Parker JDA. Disorders of affect regulation: Alexithymia in medical and psychiatric illness. Cambridge: Cambridge University Press; 1997.

6. Bruch H. Perceptual and conceptual disturbances in anorexia nervosa. Psychosom Med. 1962;24:187-94

7. Selvini-Palazzoli M. Self-starvation: from individual to family therapy in the treatment of anorexia nervosa. New York: Jason Aronson; 1985.

8. Strober M. Disorders of the self in anorexia nervosa: an organismic developmental paradigm. In: Johnson C, editor. Psychodynamic treatment of anorexia nervosa and bulimia. New York: Guilford Press; 1991. p. 354-73.

9. Becker-Stoll F, Gerlinghoff M. The impact of a four-month day treatment programme on alexithymia in eating disorders. Eur Eat Disord Rev. 2004;12:159-63.

10. Overton A, Selway S, Strongman K, Houston M. Eating disorders - The regulation of positive as well as negative emotion experience. J Clin Psychol Med Settings. 2005;12:39-56.

11. Sifneos PE. Psychosomatic, alexithymie et neurosciences. Rev Fran Psychosom. 1995;7:27-34.

12. Taylor GJ. The alexithymia construct: conceptualization, validation, and relationship with basic dimensions of personality. New Trends Exp Clin Psychiatr. 1994;10:61-74.

13. Nemiah JC, Freyberger $\mathrm{H}$, Sifneos PE. Alexithymia: a view of the psychosomatic process. In: Hill 0, editor. Modern trends in psychosomatic medicine. London: Butterworths, 1976. p. 430-9.

14. Bourke MP, Taylor GJ, Parker JDA, Bagby RM. Alexithymia in women with anorexia nervosa: A preliminary investigation. Br J Psychiatry. 1992;161:240-3.

15. Cochrane CE, Brewerton TD, Wilson DB, Hodges EL. Alexithymia in eating disorders. Int J Eat Disord. 1993;14(2):219-22. 
16. Corcos M, Guilbaud O, Speranza M, Paterniti S, Loas G, Stephan P, et al. Alexithymia and depression in eating disorders. Psychiatry Res. 2000;93(3):263-6.

17. Dethieux JB, Hazane F, Dounet C, Gentil V, Raynaud JP. À la recherche des émotions perdues: I'adolescente anorexique et son père. Étude préliminaire à propos de l'hypothèse alexithymique. Neuropsychiatr Enfance Adolesc. 2001;49:131-40.

18. Eizaguirre AE, Cabezón AOS, Alda I0, Olariaga LJ, Juaniz M. Alexithymia and its relationship with anxiety and depression in eating disorders. Pers Individ Dif. 2004;36:321-31.

19. Råstam M, Gillberg C, Gillberg IC, Johansson M. Alexithymia in anorexia nervosa: a controlled study using the 20-item Toronto Alexithymia Scale. Acta Psychiatr Scand. 1997:95(5):385-8.

20. Schmidt U, Jiwany A, Treasure J. A controlled study of alexithymia in eating disorders. Compr Psychiatry. 1993;34(1):54-8.

21. Speranza M, Corcos M, Loas G, Stéphan P, Guilbaud 0, Perez-Diaz F, et al. Depressive personality dimensions and alexithymia in eating disorders. Psychiatry Res. 2005;135(2):153-63.

22. Taylor GJ, Parker JDA, Bagby RM, Bourke MP. Relationships between alexithymia and psychological characteristics associated with eating disorders. J Psychosom Res. 1996;41(6):561-8.

23. Fernandes N, Tomé R. Alexitimia. Rev Port Psicoss. 2001;3:97-115

24. Salminen JK, Saarijärvi S, Äärelä E, Toikka TB, Kauhanen J. Prevalence of alexithymia and its association with sociodemographic variables in the general population of Finland. J Psychosom Res. 1999;46(1):75-82.

25. Honkalampi K, Hintikka J, Transkanen A, Lehtonen J, Viinamäki H. Depression is strongly associated with alexithymia in the general population. J Psychosom Res. 2000;48(1):99-104.

26. Horton PC, Gewirtz H, Kreutter KJ. Alexithymia - State and trait. Psychother Psychosom. 1992:58(2):91-6

27. Kokkonen P, Karvonen JT, Veijola J, Läksy K, Jokelainen J, Järvelin M- R, et al. Prevalence and sociodemographic correlates of alexithymia in a population sample of young adults. Compr Psychiatry. 2001;42(6):471-6.

28. Montebarocci 0 , Codispoti M, Surcinell P, Franzoni E, Baldaro B, Rossi N. Alexithymia in female patients with eating disorders. Eat Weight Disord. 2006;11(1):14-21.

29. Sexton MC, Sunday SR, Hurt S, Halmi KA. The relationship between alexithymia, depression, and Axis II psychopathology in eating disorder inpatients. Int I Eat Disord. 1998;23(3):277-86
30. Zonnevylle-Bender MJS, van Goozen SHM, Cohen-Kettenis PT, van Elburg A, van Engeland H. Emotional functioning in adolescent anorexia nervosa patients: A controlled study. Eur Child Adolesc Psychiatry. 2004;13(1):28-34.

31. Zwaan M, Biener D, Bach M, Wiesnagrotzki S, Stacher G. Pain sensitivity, alexithymia, and depression in patients with eating disorders: Are they related. J Psychosom Res. 1996;41(1):65-70.

32. Bagby RM, Parker JDA, Taylor GL. The Twenty-item Toronto Alexithymia Scale - I. Item selection and cross-validation of the factor structure. J Psychosom Res. 1994;38(1):23-32.

33. Lane RD, Sechrect L, Reidel R. Sociodemographic correlates of alexithymia. Compr Psychiatry. 1998;39(6):377-85.

34. American Psychiatric Association. Diagnostic and statistical manual of mental disorders. 4th ed. Washington, DC: American Psychiatric Association; 1994.

35. Kutlesic V, Williamson DA, Gleaves D, Barbin J, Murphy-Eberenz K. The interview for diagnosis of eating disorders-IV: Application to DSM-IV diagnostic criteria. Psychol Assess. 1998;10:41-8.

36. Torres S, Guerra MP, Lencastre L, Williamson DA, Roma-Torres A, Vieira F. Contribuição para o estudo da adaptação portuguesa da Entrevista de Diagnóstico das Perturbaçōes do Comportamento Alimentar - IV (IDED-IV) específica para a Anorexia Nervosa. Arq Med. 2008;22 (4/5):113-9.

37. Carmo I, Reis D, Varandas P, Bouça D, Padre Santo D, Neves A, et al. Epidemiologia da anorexia nervosa: Prevalência da anorexia nervosa em adolescentes do sexo feminino nos distritos de Lisboa e Setúbal. Acta Med Port. 2001;14:301-16.

38. Prazeres N, Parker J, Taylor G. Adaptação Portuguesa da Escala de Alexitimia de Toronto de 20 Itens (TAS-20). Rev Iberoam Diag E Psic (RIDEP). 2000;9:9-21.

39. Grabe HJ, Spitzer C, Freyberger HJ. Alexithymia and the temperament and character model of personality. Psychother Psychosom. 2001;70(5):261-7.

40. Laquatra TA, Clopton JR. Characteristics of alexithymia and eating disorders in college women. Addict Behav. 1994;19(4):373-80

41. Pasini A, Delle Chiaie R, Seripa S, Ciani N. Alexithymia as related to sex, age, and educational level: Results of the Toronto alexithymia Scale in 417 normal subjects. Compr Psychiatry. 1992;33(1):42-6.

42. Speranza M, Loas G, Wallier J, Corcos M. Predictive value of alexithymia in patients with eating disorders: A 3-year prospective study. J Psychosom Res. 2007;63(4):365-71.

Apêndice - Estudos de prevalência da alexitimia na anorexia nervosa

\begin{tabular}{|c|c|c|c|}
\hline Referência bibliográfica & Material & $n$ & Prevalência de alexitimia \\
\hline Bourke, Taylor, Parker, \& Bagby ${ }^{14}$ & TAS & $\begin{array}{c}\text { AN }(P)=48 \\
\text { Controle }(P)=30\end{array}$ & $\begin{array}{l}77,1 \% \\
6,7 \%\end{array}$ \\
\hline Cochrane, Brewerton, Wilson, \& Hodges ${ }^{15}$ & TAS & $\begin{array}{l}A N-R(+)=19 \\
A N-B(+)=18\end{array}$ & $\begin{array}{l}63,0 \% \\
61,0 \%\end{array}$ \\
\hline Corcos et al. ${ }^{16}$ & TAS-20 & $\begin{array}{c}\mathrm{AN}(P)=32 \\
\text { Controle }(\text { (P) }=74\end{array}$ & $\begin{array}{l}56,3 \% \\
12,2 \%\end{array}$ \\
\hline Dethieux, Hazane, Dounet, Gentil, \& Raynaud ${ }^{17}$ & TAS & 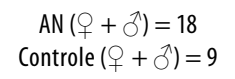 & $\begin{array}{l}55,6 \% \\
22,2 \%\end{array}$ \\
\hline Eizaguirre, Cabezón, Alda, Olariaga, \& Juaniz ${ }^{18}$ & TAS-20 & $\begin{array}{c}A N-R(q)=25 \\
A N-B(+)=44 \\
\text { Controle }(q)=43\end{array}$ & $\begin{array}{c}56,0 \% \\
68,2 \% \\
2,3 \%\end{array}$ \\
\hline Råstam, Gillberg, Gillberg, \& Johansson ${ }^{19}$ & TAS-20 & 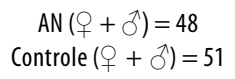 & $\begin{array}{l}22,9 \% \\
5,9 \%\end{array}$ \\
\hline Schmidt, Jiwany, \& Treasure 20 & TAS & $\begin{array}{c}A N-R(P)=55 \\
A N-B(P)=25 \\
\text { Controle }((Q)=48 \\
\text { Controle }\left(\delta^{\top}\right)=47\end{array}$ & $\begin{array}{l}56,0 \% \\
48,0 \% \\
27,0 \% \\
19,0 \%\end{array}$ \\
\hline Speranza et al..$^{21}$ & TAS-20 & $\begin{array}{c}\text { AN-R }(\&)=105 \\
\text { AN-B }(+)=49 \\
\text { Controle }(\text { (q) }=279\end{array}$ & $\begin{array}{l}56,2 \% \\
51,0 \% \\
22,2 \%\end{array}$ \\
\hline Taylor, Parker, Bagby, \& Bourke 22 & TAS-20 & $\begin{array}{c}\mathrm{AN}(P)=48 \\
\text { Controle }(+)=30\end{array}$ & $\begin{array}{c}68,8 \% \\
3,3 \%\end{array}$ \\
\hline
\end{tabular}

TAS: Toronto Alexithymia Scale (Taylor et al., 1988); TAS-20: Toronto Alexithymia Scale - 20 items (Bagby, Parker, \& Taylor, 1994); AN: participantes com diagnóstico de anorexia nervosa; AN-R: participantes com diagnóstico de anorexia nervosa, subtipo restritivo; AN-B: participantes com diagnóstico de anorexia nervosa, subtipo de ingestão compulsiva/purgativo; Controle: participantes do Grupo Controle. 